\title{
marges Marges
}

revue d'art contemporain Revue d'art contemporain

$01 \mid 2003$

Varia

\section{Voyage du signe}

Journey of the Sign

\section{Yves Bergeret}

\section{OpenEdition}

\section{Journals}

Édition électronique

URL : http://journals.openedition.org/marges/817

DOI : $10.4000 /$ marges. 817

ISSN : 2416-8742

\section{Éditeur}

Presses universitaires de Vincennes

\section{Édition imprimée}

Date de publication : 15 mars 2003

Pagination : $31-36$

ISBN : 978-2-84292-245-0

ISSN : 1767-7114

Référence électronique

Yves Bergeret, « Voyage du signe », Marges [En ligne], 01 | 2003, mis en ligne le 15 mars 2004, consulté le 07 mai 2019. URL : http://journals.openedition.org/marges/817 ; DOI : 10.4000/ marges.817 


\section{Voyage du signe}

Dans la pénombre chaude de sa maison, Hama a pris dans sa main de la terre ocre mêlée d'eau ; l'eau de la source, là-haut dans les rochers, Téga la source où seules les femmes se rendent pour rapporter dans de grandes calebasses sur leur tête l'eau au village. Hama a pris la terre, l'a mêlée d'eau, l'a malaxée.

Dans la pénombre chaude, Hama a gardé juste un peu de la terre ocre au bout de trois doigts qu'il a serrés ensemble. Et la terre ocre humide, il l'a posée longuement fermement sur le mur en banco droit devant lui, qui tourne le dos à la porte par où un peu de la lumière brutale du jour afflue. Son épaule, son bras, ses doigts prolongent le jour jusqu'au mur qu'il touche. Et voici, de ses trois doigts il dessine à l'ocre sur le mur beige une sorte de silhouette sans doute humaine. Puis il reprend de la terre, la mouille et, le bras droit devant lui, il installe le contour d'un grand carré vide, puis, encore, ici le contour et le contenu opaque d'un autre carré, et encore un carré ; cela fera ce soir un damier. Et ici, il demande à sa femme la petite demi-calebasse qui sert de louche, il l'applique au mur et de ses trois doigts il en parcourt le contour et laisse la trace ocre de son contour sur le mur. Contour ocre : ainsi vient au monde le signe graphique. Vient au monde, sur le mur face à la porte d'entrée, signe comme des clignements d'œil devant la lumière du jour, comme des bulles d'air échappées du fond des eaux, comme une émergence ici, sur ce mur sombre, du début d'une langue écrite. 
Hama, et d'autres paysans comme lui dans ce village, et d'autres encore dans d'autres villages, peignent à l'intérieur de chez eux. J'ai vu leurs peintures, qu'ils m'ont montrées ; je séjournais dans le village pour la troisième fois, ayant déjà travaillé avec les grands élèves de l'École, dans un atelier d'écriture que j'animais. Avant la tombée de la nuit, j'allais voir les peintures qu'on voulait bien me montrer, toutes plus belles les unes que les autres, plus intrigantes aussi dans leur fraîcheur (impudeur ?) droite.

La parole du poème vient du lieu où je vis. Je vis dans ce village depuis quelques jours ; je dors chez un cultivateur, à même le sol de sa cour, sous les étoiles. L'eau de la maison, c'est celle que les femmes vont chercher à la source le matin et qu'elles gardent dans une grande jarre de terre. La parole du poème, les mots qui vont être sa chair, la parole qui va être son sang et son sens, viennent par les pas des porteuses d'eau à l'aube. La parole vient par le cheminement lent du lieu, terre, roche et falaises abruptes, là-bas, de la montagne que chaque jour de son existence Hama regarde comme une seconde mère au front énigmatique.

Ainsi j'écris peu à peu le poème ; je propose à Hama que nous fassions œuvre ensemble. Oui, dit-il. Je déploie au sol une bannière de tissu uni presque blanc, un mètre de large sur deux de haut ; j'ai fait coudre un large ourlet en haut et un autre, de même, en bas, par lesquels passer une baguette de bois, plus tard, lorsque l'on suspendra l'œuvre, poème-peinture, ainsi réalisée. J'écris au crayon noir les mots de mon poème, dans une graphie ample et dans une mise en page très aérée. Hama, et bientôt vingt personnes avec lui, toutes analphabètes, toutes portées par les savoirs subtils, complexes et maintenant morcelés de l'oralité, me regardent écrire. Je repasse à la peinture noire mes lettres, labiles, cursives ; je lis les mots du poème, lentement, à voix forte. Deux, même trois et bientôt quatre personnes autour du tissu, traduisent le poème, le commentent, et chacun intervient et commente. J'ai préparé trois petits pots de peinture, avec chacun son pinceau, un bleu, un jaune et un rouge.

Hama se saisit alors d'un des pinceaux, réfléchit et, soudain, toute décision prise, sans la moindre hésitation, pose un premier trait de couleur, puis un second. Il change de pinceau et de couleur. Puis il pose encore tel trait, tel autre trait. Et ainsi, au bout de quelques heures, les signes peints sont venus sur le tissu et la bannière existe. Bannière venue de rien, non pas ; mais venue de ce que nous sommes et de ce que nous sommes parce que nous vivons en résonance incessante avec ces lieux-ci. Conversation ininterrompue 
entre Hama et moi, entre les lieux et nous, à laquelle nous donnons cette forme, la bannière poème-peinture, comme un arrêt d'étape.

Que se passe-t-il ici ? Hama et d'autres peintres qui se saisissent à leur tour du pinceau, parfois sur le même tissu que leur ami a commencé de peindre, sont, dans une sorte de jeu archétypal beau et entier comme une liturgie tragique, requis par une turbulence $d u$ sens que la métaphore du poème déclenche. Or cette turbulence est d'abord ici figurée par la graphie souple des lettres en noir. Sur le plat du tissu au sol, devant tous (et non plus à l'intérieur de la maison privée), en pleine lumière, la parole du poème fonde un monde et l'espace de celui-ci. Le tissu que j'utilise devient un carrefour, carrefour des regards de tous, carrefour de ma main qui écrit et des mains de ceux qui peignent, carrefour du sens qui s'élance ou même s'égare dans une aventure d'une fraîcheur extraordinaire. Le peintre pose ici non pas un et un trait. Mais il pose ce qui devient l'échafaudage d'un signe, dont la signification lui est totalement explicite et claire : une maison, tel animal, tel arbre, tel outil pour le jardinage, telle autre maison de pierre ou de branchage. Le monde revient à la course danser non pas autour mais avec les mots du poète. Et s'il danse, c'est qu'il a, par notre conversation ininterrompue, pris soudain une vie oblique où les profils et les silhouettes se forment comme jamais jusqu'ici.

Par la forme du support, il se produit déjà, au sens exact, un événement neuf. L'image que constituent les traits et les contours de couleur qu'ont posés les paysans-peintres s'impose d'abord au regard comme un tout : une image globale, tel un vaste idéogramme dont les clefs nous échapperaient, à nous Occidentaux ; mais sans doute pas aux paysans-peintres ni à leurs proches dans les villages, tant le besoin du signe graphique est maintenant une réalité quotidienne, alors que s'effacent les savoirs ancestraux de l'oralité. De plus, les paysans-peintres, fort souvent, posent sur le tissu, évidemment hors toute perspective renaissante, parfois en axonométrie, parfois selon des hiérarchies visuelles complexes dont l'organisation m'échappe encore, la silhouette d'objets ou d'animaux, voire de personnages qui leur sont familiers. C'est un monde saisi et refait qui se donne ainsi, en vision globale immédiate ; on peut ensuite aller librement de tel à tel détail peint.

Mais les mots de mon poème nécessitent, par le fait même de la lecture lettre à lettre, une saisie lente et linéaire, non pas globale ; de plus ma graphie parfois assez libre, mais toujours lisible, ralentit 
une saisie immédiate. Enfin l'usage d'une métaphore simple et, de ce fait, sans doute plus ouvrante (j'ose ce terme) encore, suspend toute vision immédiate. Si bien que la bannière poème-peinture est un carrefour aussi : elle croise une vision frontale, horizontale et plate, celle des signes peints, avec une vision perpendiculaire à la précédente, celle des mots du poème, échappée verticale dans l'épaisseur étrange du monde. Cette bannière n'est pas en deux dimensions, elle est en multiples dimensions. Cela, les paysanspeintres l'ont compris d'emblée et l'apprécient d'autant plus qu'une réalisation de bannière est également une liturgie ludique, tous à genoux au sol, dans une conversation de tous les acteurs et de tous les témoins, avec une joie grave et heureuse et, enfin, au bout de la dramaturgie simple et lumineuse, nous dressons la bannière, debout en plein vent.

Et ainsi sur le mur extérieur d'une maison du village, ou sur le rocher sacré qui surplombe la source, ou sur la branche basse du baobab, se trouve accomplie la construction où se rencontrent le mot et le signe graphique, carrefour frais et hardi de significations hypothétiques, heureuses, jetées en plein vent, dehors, en pleine lumière, au regard de tous. Aventure du signe.

Puis avec l'accord et même le souhait des paysans-peintres, la bannière commence avec moi son long et lent voyage, vers d'autres villages plus loin dans ces montagnes du désert, où ils savent que je la suspends parfois, en compagnie des nouvelles bannières créées dans tel ou tel nouveau village ; plus tard à Bamako ; bientôt en France et en Europe, où je montre les bannières dans des conférences ou des expositions ; et ainsi, un jour, jusqu'en Haïti, où ces bannières maliennes sont allées rencontrer celles que je créais à Port-au-Prince et dans la montagne au-dessus de la ville avec des peintres artisans charpentiers et maçons.

Les paysans-peintres des montagnes désertiques ne se sentent pas dépossédés des signes qu'ils ont posés sur les tissus qui voyagent ; au contraire, me disent-ils, ils confient à mes mots la compagnie de leurs signes; et ainsi part pour un lointain voyage, qu'eux-mêmes n'accompliront peut-être jamais, cette partie de leur personne, bras neuf, troisième clavicule, œil pour la vision très lointaine, qu'ils délèguent depuis le fond de leur personne. Ils pensent que chacun d'eux voyage par le signe. Et ils me demandent que, oui, la bannière voyage et voyage et soit vue et soit présentée encore et encore. C'est une nouvelle partie errante de l'âme du paysan-peintre qui 
entreprend le grand voyage ; mais si elle le fait, c'est d'abord parce que l'égide ferme du mot écrit élance et garantit ce voyage ; et pas n'importe quel mot, mais la parole du poème ; de ce poème qui m'est venu par le dialogue avec la langue-espace du lieu. Les paysans-peintres le savent, qui ont vu, qui ont pris part à toute la gestation du poème et à celle de la bannière. Ils m'ont vu arriver dans le village, par une longue marche, parfois harassante sous le soleil impitoyable dans la pente de la montagne, sous l'harmattan qui apporte la poussière abondante du Sahara ; ils m'ont accueilli ; ils m'ont vu dormir à la belle étoile sur le sol devant leurs maisons ; et au bout de quelques jours les mots du poème sont ici, tracés à grands gestes de la main sur le tissu posé sur le sol, sur leur sol devant la porte de leur maison.

Si les poèmes-peintures sur le tissu sont clairement destinés à voyager, et chacun le sait dès le départ, il n'en va pas de même pour les « installations » de poèmes que j'écris sur des pierres. Je choisis, toujours avec les habitants d'un village, un lieu qui me semble particulièrement propice à la parole et, de plus, beau, par son architecture naturelle elle-même et par les lointains sur lesquels il donne. Souvent, j'ai déjà vu ce lieu lors d'un précédent séjour de travail, y ai pensé maintes et maintes fois, ai imaginé les poèmes qui lui conviendraient. Enfin, ce matin, après encore des jours et des jours passés dans cette montagne, dans ce village, je demande aux Anciens du village s'ils sont d'accord pour que j'écrive des poèmes sur des pierres. Une discussion parfois assez longue s'ensuit, qui toujours débouche sur un accord et nous voici déjà sur ce grand champ de dalles à peine inclinées, au pied d'une haute falaise orange, face aux plaines du Sahara ; ou, cet autre jour, au sommet de la montagne dont l'accès est commandé par un village très ancien. Je regarde, je pense au lieu où nous nous trouvons maintenant, les habitants m'en parlent.

Les mots enfin sont là, prêts à être écrits. On a choisi des pierres dont une face est plate, je les nettoie et, les genoux au sol, en plein soleil, j'écris à la peinture noire les lettres du poème. Ensuite nous dressons ces pierres, qui sont souvent cinq, comme une sorte de jardin de stèles, face à nos regards, face aussi à la grande falaise orange, sentinelles de parole et de paix devant l'horizon presque invisible du Sahara. Je lis enfin à voix haute les poèmes de ces pierres, tous lisent les mots, que certains traduisent, tous commentent.

Il m'est arrivé à Kantakine, un village au-dessus d'une petite falaise, que les habitants, à peine avais-je fini avec une « installation » de 
poèmes, me demandent d'en faire aussitôt une seconde. « Mais, je ne peux improviser ; il me faut réfléchir au lieu ; à cette "installation" que nous venons de mettre en place, j'ai pensé pendant six mois. - $\mathrm{Si}$, nous voulons que tu nous écrives des poèmes sur des pierres, là-bas, un peu plus loin ». Nous y allons. En effet le lieu est d'une grande beauté, des aiguilles rocheuses isolées, vers l'Ouest, forment au loin comme des silhouettes de stèles immenses privées de signes. Oui, j'essaye de créer une nouvelle « installation », sur trois très belles pierres, fort lourdes, que nous avons trouvées sur place ; oui, quelques heures après, l'« installation " est en place. "Elle aussi est à vous, j'ai écrit ces poèmes pour vous et pour le village de Kantakine ». Les Anciens m'expliquent qu'une double source jaillit là pendant les mois orageux de l'hivernage et qu'il est bon que la parole d'un poème soit présente à l'emplacement de la source.

En effet, si le signe graphique peint sur le tissu est fait pour voyager, le poème écrit sur la pierre est fait pour s'ancrer dans l'espace ; l'un part, l'autre reste. Si les signes emportent avec eux un peu de délégation d'âme, il est nécessaire qu'un peu de mon âme reste à jamais par ces traces écrites, sur les pierres relevées près du village. D'ailleurs lorsque je reviens, quelques mois plus tard, il arrive qu'on me demande de refaire le tracé noir des lettres car le soleil et le vent, terribles, ont déjà effacé telle ou telle lettre. Circulation du signe et de la parole, échange dynamique, ancrage et voyage ; ainsi en va-t-il de ce poème et de ces poèmes-peintures que donne la langue-espace de ces villages des montagnes du désert.

Chacun dans ces villages sait que j'habite en Europe, que la durée de mes séjours reste limitée : au plus un mois. Mais j'annonce que dans quelques mois je reviendrai ; et en effet je reviens, je retrouve mes amis peintres ; je fais parfois connaissance de nouveaux peintres, et nous reprenons sans aucune hésitation le travail de création. Trois fois, lors de mon séjour de février 2002, on m'a offert un terrain. Tu connais nos maisons de terre : tu dois t'en construire une.

Yves Bergeret 